\title{
Analytical presentation of the separation of dense suspension for the extraction of amber
}

\author{
Volodymyr Nadutyi $^{1}$, Valerii Korniienko ${ }^{2}$, Zinovii Malanchuk ${ }^{2}$, and Olha Cholyshkina ${ }^{3, *}$ \\ ${ }^{1}$ Institute of Geotechnical Mechanics named by N. Poljakov of National Academy of Sciences of \\ Ukraine, 49005, Dnipro, Simferopolska Str., 2a, Ukraine, \\ ${ }^{2}$ National University of Water Management and Nature Resources Use, 33028, Rivne, Soborna \\ Str., 11, Ukraine \\ ${ }^{3}$ PrAT "Higher Educational Institution "Interregional Academy of Personnel Management", 03039, \\ Kyiv, Frometivska Str., 2, Ukraine
}

\begin{abstract}
New devices were developed to increase the extraction of amber, especially with the size less than $5 \mathrm{~mm}$, which can not be extracted at the present time. They are made on the basis of a hydraulic spiral classifier using vibration and bubbling with air bubbles. Analytical formulas are needed to calculate such devices, in first, for free motion of particles, then the constrained motion, and then to determine the effect of vibration and bubbling on the speed. This article discussed the first part of the problem - hydrodynamics of free movement of amber and quartz particles in a mixture of amber sand-clay rocks with water. The technique and formulas of calculation of kinematic viscosity and speed of movement are given. The dependences of the kinematic viscosity and the speed of free deposition of quartz and free ascent of amber on the density of the suspension for particles size less than $5 \mathrm{~mm}$ are obtained. The non-linear character of the dependence of the speed of ascent of amber from the density of the suspension is set. The results obtained are the basis for the calculation constructional parameters and technological characteristics of the hydraulic classifiers of new construction.
\end{abstract}

\section{Introduction}

Extraction of amber in the fields of Ukrainian Polissia is mainly carried out by we hydraulic method $[1,2]$. Its disadvantages are low, up to $50 \%$, extraction of amber, and destructive impact on the ecosystem. This one, as well as other known technologies, do not provide the extraction of small fractions of amber with a size less than $5 \mathrm{~mm}$.

The methods and devices for the extraction of amber, including small $5 \mathrm{~mm}$ fraction, are developing in the Institute of Geotechnical Mechanics named by N. Poljakov of National Academy of Sciences of Ukraine together with the National University of Water Management and Nature Resources Use (Rivne) [1-4]. They provide the layer-by-layer excavation of amber-bearing rocks and subsequent extraction of amber using mechanical and hydraulic classification. Thus, on the basis of known spiral classifier, the devises have

Corresponding author: chel.valenti@gmail.com 
been developed, and they differ from the known devices by use of additional impacts vibration and bubbling of the suspension by air bubbles [3, 4]. The principle of separation is based on different deposition rate of particles of quartz and emerging of amber in the separation zone, which is limited by the surface (mirror) of the pulp in the classifier bath (Fig. 1).

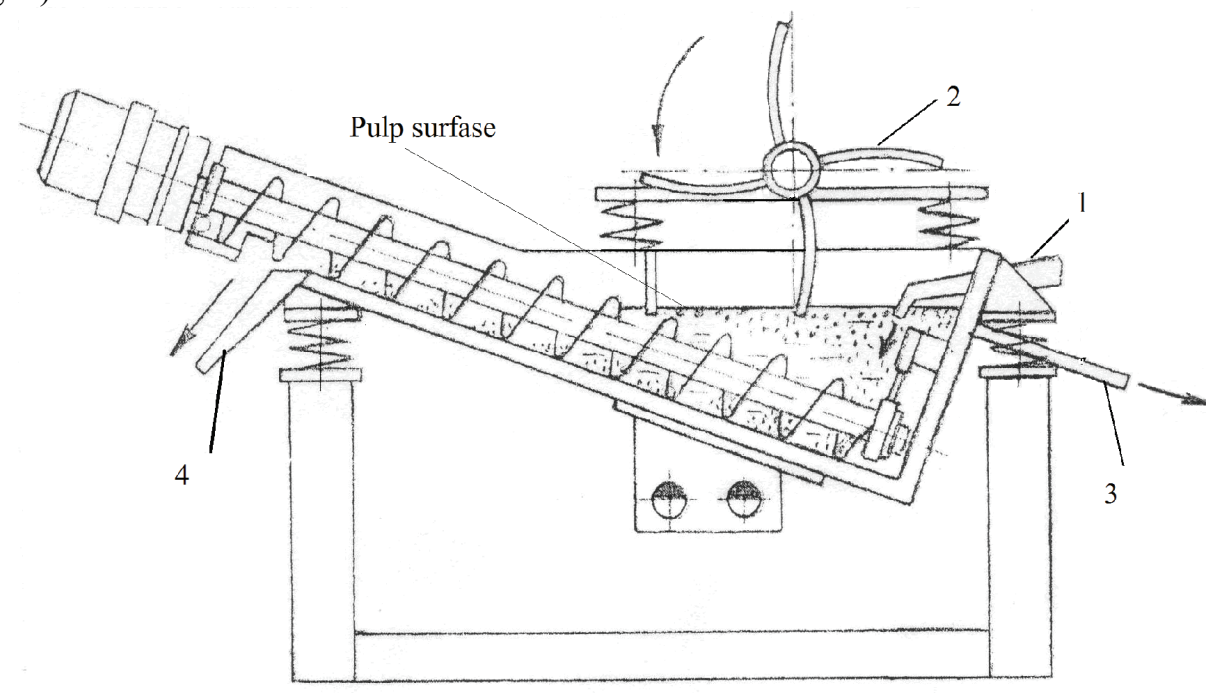

Fig. 1. Vibro-classifier of complex action for amber extraction: 1 - additional (circulating) water; 2 rowing device to move emerged fraction; 3 - discharge chute for emerged amber fraction; 4 - sand discharge.

Figure 1 shows amber extracting device, which is based on a spiral classifier. Mirror pulp is formed in a side power supply, additional and circulating (return) water (1). Perforated tubs with air supply for bubbling of the suspension is placed in the bath. A device for creating vibration oscillations is installed under the bottom of the bath. By means of a rotary rowing mechanism (2), amber is unloaded into the receiving chute (3), the sands are unloaded in the upper zone of the classifier (4).

In the theoretical analysis of the process of hydraulic separation of particles, firstly, the speed of free, and secondly, the speed of constrained motion - deposition or ascent - is determined. Semi-empirical formulas or empirical (experimental) coefficient of transition from the speed of free movement to the speed of the constrained movement has been used for constrained motion. This article deals with a particular issue of free movement of single particles of quartz and amber in dense suspensions.

The calculation of hydrodynamics use kinematic viscosity, the value of which at equivalent measurements of different authors has a large spread.

The aim of the work was to establish the dependences and calculation formulas for determining of the rate of free deposition of quartz particles and the ascent of amber, and determining the kinematic viscosity of the suspension in the form of a mixture of amberbearing rock with water of different densities, where particle size is less than $5 \mathrm{~mm}$.

\section{Methodology}

The method of work consists in using well-known hydrodynamic methods to obtain sound mathematical formulas for calculating the processes of free ascent of small amber particles and free precipitation of quartz in the separation of dense pulp in the working area of the vibroclassifier. 
The supply of vibro-classifier is amber-bearing rocks of Klesovskyi deposit. The average amber content is 19-21 g/t [5]. Enclosing rocks consist of fine and medium-sized sand with a small amount of clay. According to water-slurry scheme, the density of the suspension (pulp) in the classifier bath can be $1.4-1.7 \mathrm{~g} / \mathrm{cm}^{3}$.

Amber density is $1.05-1.24 \mathrm{~g} / \mathrm{cm}^{3}$ [5]. In calculations accepted $\rho_{a m b}=1.24 \mathrm{~g} / \mathrm{cm}^{3}$, which will allow to determine the lower limit of the rate of amber ascent, due to the less dense particles will float faster. Since the amber content in the rock is not high, the main component is sand, then in fact, the density of the solid phase of suspension is the density of quartz $\rho_{q u a}=2.65 \mathrm{~g} / \mathrm{cm}^{3}$. Since the density of amber is less than the density of the liquid phase of the suspension (water), then we do not calculate the deposition rate for amber, but the ascent rate for amber.

\section{Results and discussion}

With the increase of the dispersed phase in the suspension, the interaction of the particles increases, but as long as they do not form a constant structure, then suspension can be considered a Newtonian liquid. The Physical state of the pulp in the classifier does not reveal the presence of a structure, in which the particles are oriented relative to each other in a certain way.

This means that this system "liquid - solid" is a poly-disperse, not structured Newtonian fluid, and for this system we can use Newtonian fluids formulas of viscosity. Among the latter, the most famous formula is Einstein formula, as well as its clarifying formula of L.D. Landau [6]. They are valid at volume concentration of solid $\beta=2-5 \%$. Zuber, Vakhrushev and Wend formulas in the form of a power polynomial in $\beta$ - are valid if concentration of solid is less than $25 \%$.

The widest range of the solid concentration in the suspension is described by the Wend formula, which is in good agreement with the experimental data for all values of the suspension's concentrations, at which the fluidity of the system is still maintained [6]:

$$
v=v_{0} \exp \frac{2.5 \cdot \beta+0.675 \cdot \beta^{2}}{1-0.609 \cdot \beta}
$$

where $v_{0}=0.01 \mathrm{~cm}^{2} / \mathrm{s}$ - kinematic viscosity of water at $20{ }^{\circ} \mathrm{C},[9] ; \beta$ - the volume fraction of solid or the coefficient of the volume concentration of solid in suspension.

The volume fraction of solid in suspension $\beta$ is defined as:

$$
\beta=\frac{Q_{\text {sol }}}{Q_{\text {sol }}+Q_{\text {lic }}}, \quad Q_{\text {sol. }}=\frac{T_{\text {sol }}}{\rho_{\text {sol }}}, \quad Q_{\text {lic }}=\frac{T_{l i c}}{\rho_{\text {lic }}}
$$

where $Q_{l i q}, Q_{\text {sol }}$ - the volume of liquid and the volume of solid in the pulp, respectively; $T_{\text {liq }}, T_{\text {sol }}$ - the load on the classifier on the water and solid, is determined by the water-slurry scheme.

Program of calculation is developed for building of the technological scheme.

For example, at productivity of the vibro-classifier $10 \mathrm{t} / \mathrm{hr}$ load on the device, taking into account the circulation, was: $T_{\text {sol }}=11.5 \mathrm{t} / \mathrm{hr}$, water: $T_{\text {liq }}=7.61 \mathrm{t} / \mathrm{hr}$.

Here with, present of solid in the bath was $60.2 \%$, density $\rho=1.6 \mathrm{~g} / \mathrm{cm}^{3}$ or $1600 \mathrm{~g} / \mathrm{l}$.

Accordingly: $Q_{l i q}=7.61 \mathrm{~m}^{3} / \mathrm{hr}, Q_{\text {sol }}=11.5 / 2.65=4.3 \mathrm{~m}^{3} / \mathrm{hr}$.

The coefficient of the volume concentration of solid $\beta=0.363$.

Kinematic viscosity of the suspension according to the formula (1) is $v=0.036 \mathrm{~cm}^{2} / \mathrm{s}$. 
On the basis of formulas (1), (2), taking into account that for water $v=0.001 \mathrm{~cm}^{2} / \mathrm{s} \mathrm{at}$ $20^{\circ} \mathrm{C}$, the dependence of kinematic viscosity change on the suspension density is obtained (Fig. 2).

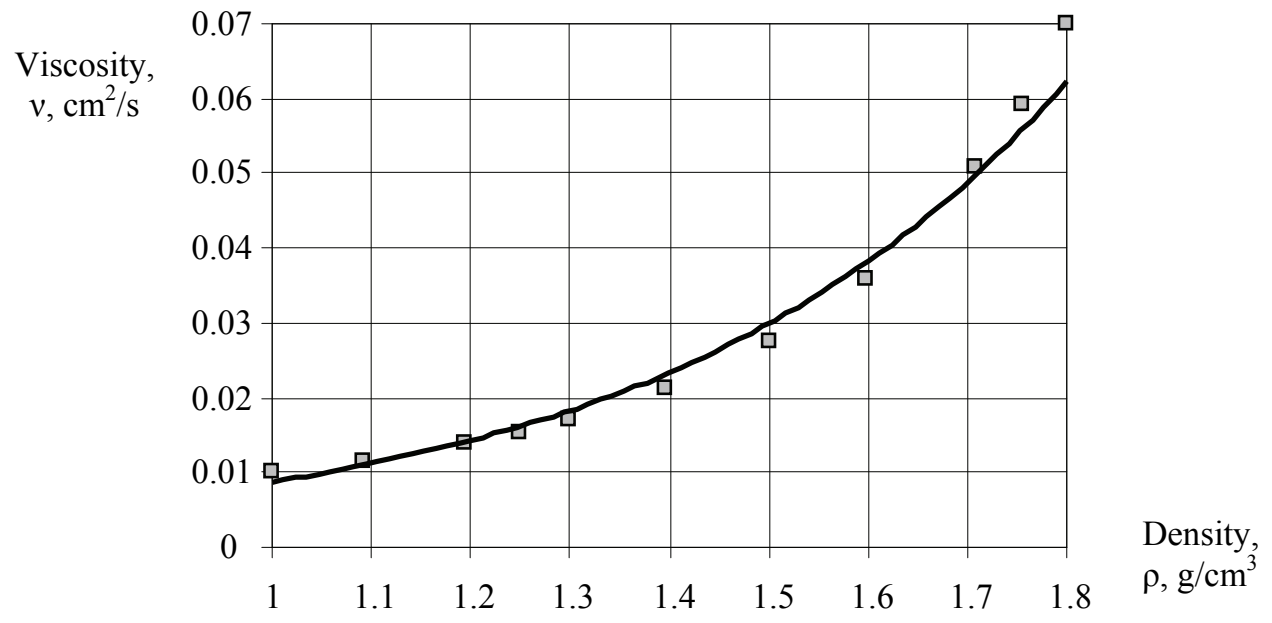

Fig. 2. Dependence of the kinematic viscosity on the pulp density.

The interpolation equation for determination of kinematic viscosity of amber-bearing pulps of different density with a high adequacy, in terms the square of correlation coefficient $R^{2}$, have a form:

$$
v=0.0008 \cdot e^{2.455 \cdot \rho}, \quad R^{2}=0.986
$$

Formula (3), unlike (1), depends only on the pulp density in the classifier bath, which is easily measured in production conditions, for example, by weighing of measuring one-liter mug with pulp.

To calculate the speed of free deposition of particles on the diagram of Rayleigh (dependence of the particle deposition resistance coefficient on the Reynolds number) there are several areas, each of which is interpolated by its calculation formula (Table 1), [6].

Table 1. The speed of free deposition of particles in the liquid, depending on the criteria $A$, [6] and criteria of Reynolds: $R e=V \cdot d / v,\left(d-\mathrm{cm}, v-\mathrm{cm}^{2} / \mathrm{s}\right)$.

\begin{tabular}{|l|l|l|}
\hline \multicolumn{1}{|c|}{ Area on Rayleigh curve/author } & \multicolumn{1}{|c|}{ Criteria: $A, R e$} & \multicolumn{1}{c|}{ Speed, $\mathrm{cm} / \mathrm{s}$} \\
\hline Laminar / Stokes & $0<A<5.25, R e<0.5$ & $V=54.481 \cdot d^{2} v^{-1} \cdot \Delta$ \\
\hline $\begin{array}{l}\text { The beginning of the transition } \\
\text { area }\end{array}$ & $5.25<A<720,0.5<R e<30$ & $V=23.6 \cdot d^{3 / 2} \cdot v^{-2 / 3} \cdot \Delta^{5 / 6}$ \\
\hline Middle / Allen & $720<A<23000,30<R e<300$ & $V=24.3 \cdot d^{-1 / 3} \cdot \Delta^{2 / 3}$ \\
\hline The end of the transition area & $\begin{array}{l}23000<A<1.4 \cdot 10^{6} \\
300<R e<3000\end{array}$ & $V=37.2 \cdot d^{2 / 3} \cdot v^{-1 / 9} \cdot \Delta^{5 / 9}$ \\
\hline Turbulent / Newton - Rittinger & $\begin{array}{l}1.4 \cdot 10^{6}<A<1.7 \cdot 10^{9} \\
R e>3000\end{array}$ & $V=57.5 \cdot d^{1 / 2} \cdot \Delta^{1 / 2}$ \\
\hline
\end{tabular}

The choice of the calculation formula we performed not according to the Reynolds criterion $R e=V \cdot d / v$, since it includes two uncertain values - the speed and diameter of the particle, but using dimensionless criterion A [6]:

$$
A=\frac{\pi \cdot d^{3} \cdot g \cdot \Delta}{6 \cdot v^{2}}
$$




$$
\Delta=\frac{\rho_{s o l}-\rho_{l i c}}{\rho_{l i c}}
$$

where $d$ - diameter of the particles, $\mathrm{cm} ; g$ - acceleration of gravity (free fall acceleration), $981 \mathrm{~cm} / \mathrm{s}^{2} ; \rho_{\text {sol }}, \rho_{\text {liq }}-$ density of solid and pulp, accordingly, $\mathrm{g} / \mathrm{cm}^{3} ; v-$ kinematic viscosity, $\mathrm{cm}^{2} / \mathrm{s}$.

The method of speed calculation is as follows:

- we define the limits of the particle size. To do this, we give the value $A$ limit's values and we find the corresponding values $d$ from the formula (4);

- after we specify $d$ by the limit's values $R e$ (Table 1 ). We determine the viscosity of the suspension by the formula (3);

- we set the desired particle size $d_{0}$. Depending on which of the above-mentioned limits of size falls $d_{0}$, we select the formula for calculating the speed in Table 1 .

- using Microsoft ${ }^{\circledR}$ Excel ${ }^{\circledR}$ program, we calculate speed for the particle $d_{0}$ within these limits, and perform a graphical construction and select the most reliable approximating equation of the trend line.

In accordance with this method, we substitute in the equation (4) limit values $A$ from Table 1 and valuevby formula (3) at pulp density 1.4 and $1.6 \mathrm{~g} / \mathrm{cm}^{3}$. For amber in formula (5) we take the modal value $\Delta$ and take into account, that we then determine the speed of ascent rather than speed of deposition. It is found, that for particles of given size - less than $5 \mathrm{~mm}$, we need to use two formulas-Allen's formula and formula of the beginning of the transition area. For the given conditions, the computer capabilities allowed to obtain simple interpolation formulas for calculating the speed (Table 2).

Table 2. The speed of deposition of quartz and ascent of amber of small size classes the density of the suspension 1.4 and $1.6 \mathrm{~g} / \mathrm{cm}^{3}$.

\begin{tabular}{|c|c|c|}
\hline \multicolumn{2}{|c|}{ Quartz } \\
\hline \multirow{2}{*}{1.4} & $0.17<d<0.88$ & Speed $V=f(d), \mathrm{mm} / \mathrm{s}$ \\
\hline \multirow{2}{*}{1.6} & $0.88<d<2.81$ & $89.21 \cdot d^{1.5}$ \\
\cline { 2 - 3 } & $0.21<d<1.4$ & $81.67 \cdot d$ \\
\hline \multirow{2}{*}{1.6} & $1.4<d<4.5$ & $48.188 \cdot d^{1.5}$ \\
\hline \multirow{3}{*}{1.4} & Amber & $55.58 \cdot d$ \\
\hline \multirow{3}{*}{1.6} & $0.3<d<1.76$ & $19.249 \cdot d^{1.5}$ \\
\cline { 2 - 4 } & $1.76<d<5.57$ & $20.74 \cdot d$ \\
\hline \multirow{2}{*}{} & $0.4<d<2.0$ & $19.748 \cdot d^{1.5}$ \\
\cline { 2 - 4 } & $2.0<d<6.4$ & $27.22 \cdot d$ \\
\hline
\end{tabular}

The dependence of the speed of free motion of particles from the pulp density shows that the deposition speed of quartz decreases linearly with increasing of density (Fig. 3).

Both - quartz and amber - move in a medium with the same viscosity, but the nature of the amber speed is radically different form the quartz speed, it is nonlinear depending on the density of the suspension (Fig. 3). This dependence is approximated by a polynomial of the second degree (square) with the accuracy of the approximation not lower $R^{2}=0.98$. An increase of the suspension density above certain values does not lead to an increase of 
ascent speed of amber; on the contrary, there is decrease in it. The authors made the choice of rational density range later, based on the analysis of constrained motion, which is not included in the scope of this article.

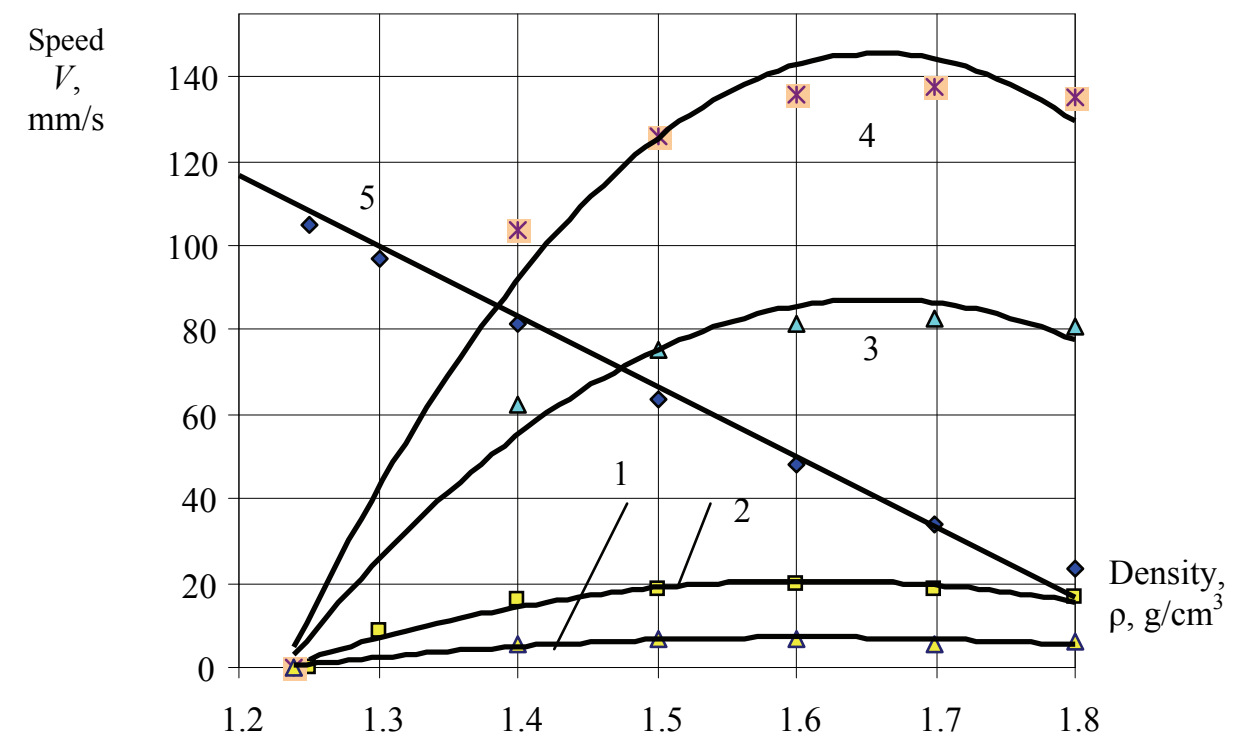

Fig. 3. Speed of free motion of particles from the pulp density: ascent of amber with size: $1-0.5 ; 2-$ $1.0 ; 3-3.0 ; 4-5.0(\mathrm{~mm}) ; 5$ - deposition of quartz with size $1 \mathrm{~mm}$.

As follows from Figure 3, with the increase of amber size the speed of ascent increases. According to the degree of influence of the particle speed, the factors are arranged in the following order: particle density, size, medium density, viscosity. This shows the analysis of regression coefficients built using the application package of statistical data processing SPSS Statistics. The obtained hydrodynamic characteristics of the viscosity of the suspension and speed of free deposition of quartz particles and ascent of amber are the basis for calculating the speed of constrained motion and further calculation of the design and technological characteristics of devices, for example, particle size in the drain of the classifier. The presented method of calculation is suitable for both spiral and other types of hydraulic classifiers. When calculating the design characteristics of the classifier, the obtained hydrodynamic characteristics of both, quartz and amber, are used, since they enter into drain of the classifier together.

\section{Conclusions}

Here described the methods, calculation formulas, dependence of kinematic viscosity and the speed of free deposition of quartz and ascent of amber on the density of the medium for particle size led then $5 \mathrm{~mm}$ for suspension in the form of a mixture of amber-bearing rocks with water.

The nonlinear character of the dependence of the amber ascent speed on the suspension density is established.

The results obtained are basis for calculating the speed of the constrained particle motion and its correction taking into account the influence of vibration and bubbling of the suspension. This speed is an important characteristic. At a given performance of the classifier, it allows to calculate the design parameters and performance characteristics, in 
particular, water consumption for the process of separation of particles of a given size in hydraulic classifier.

\section{References}

1. Bulat A.F., Nadutyy V.P., Malanchuk E.Z., Malanchuk Z.R., Kornienko V.Ya. (2017). Promyslovi tehnologii vydobutku burshtynu [Industrial technologies for the production of amber], Dnipro-Rivne

2. Z. Malanchuk, V. Korniyenko, Modern condition and problems of extraction of amber in Ukraine , Canadian Journal of Science and Education. 2, (2014)

3. Nadutyy V.P., Malanchuk Z.R., Chelyshkina V.V., Korniyenko V.Ya. (2017). Sposib viluchennya burshtinu [Aamber extraction method], Patent No 120248, Ukraine

4. Nadutyy V.P., Chelyshkina V.V., Suhariev V.V., Korniyenko V.Ya. (2015), Vibratcziynyy klasifikator [Vibration Classifier], Patent No 102869, Ukraine

5. Srebrodolsky B.I. (1980). Yantar Ukrainy [Amber of Ukraine]. Kyiv: Naukova dumka, 29-31

6. Bogdanov O.S., Olevsky V.A., Akinshin I.K., Baschenko N.T. (1972). Spravochnik po obogascheniju rud [Handbook for ore enrichment], 1, Moskva: Nedra 